\title{
All that is Miliary is not tuberculosis
}

\author{
Vijetha S ${ }^{1}$, Samanvitha V ${ }^{1}$, Ramu M ${ }^{2}$, Satish Chandra K ${ }^{3}$, Prasad C N ${ }^{4}$.
}

1. Junior Resident, Department of Respiratory medicine, Prathima institute of medical sciences, Karimnagar, Telangana.

2.Assistant Professor, Department of Respiratory medicine, Prathima institute of medical sciences, Karimnagar, Telangana. 3. Head of the department, Department of Respiratory medicine, Prathima institute of medical sciences, Karimnagar, Telangana. 4. Professor, Department of Respiratory medicine, Prathima institute of medical sciences, Karimnagar, Telangana.

Corresponding author: Vijetha S; E-mail address: vijethareddy93@gmail.com

Department of Respiratory Medicine, Prathima institute of medical sciences, Karimnagar.

E-mail address: vijethareddy93@gmail.com (Vijetha S), Mobile number:9052249568.

(C) 2020-21 Prathima Institue of Medical Sciences

\begin{abstract}
Miliary pattern on radiographs are attributed always to tuberculosis even though the differential diagnoses of miliary pattern is very wide. This pattern is most commonly caused by infectious diseases and rarely by malignancies. Primary lung cancers presenting with miliary shadows is extremely rare.

Here we report a case of 50-year old female, presenting with 15 days of symptoms and CXR PA view showing bilateral diffuse miliary nodules. HRCT Chest confirmed the miliary pattern and also showed a mass in left lower lobe. Microbiological tests for tuberculosis and fungal infections were negative. CECT Chest gave impression of left lower lobe suspected malignant lung mass with pulmonary metastasis. Malignancy was confirmed by CT guided FNAC as Adenocarcinoma lung. PET scan didnot reveal metastasis in other organs.
\end{abstract}

\section{CONCLUSION :}

Miliary pattern does not always indicate tuberculosis and other possibilities should be evaluated.

KEY WORDS: Miliary pattern, adenocarcinoma, CT guided biopsy, PET CT scan.

\section{INTRODUCTION}

The term nodule is used to describe a rounded opacity in lung parenchyma on HRCT Chest which is less than $3 \mathrm{~cm}$ in diameter and a micronodule as an opacity less than $3 \mathrm{~mm}$ in diameter whereas Miliary nodules are interstitial opacities of 1-2 mm diameter. ${ }^{1}$

On a Chest X-Ray an opacity of $2-3 \mathrm{~mm}$ is visible as a miliary shadow. ${ }^{2}$ These miliary opacities are random nodules with no defined distribution like peri-lymphatic or centrilobular pattern.

There are varied differentials that may appear as miliary opacities on Chest X-ray such as tuberculosis, histoplasmosis, coccidiodomycosis, cryptococcosis, blastomycosis, sarcoidosis, coal miners pneumoconiosis, silicosis, haemosiderosis, langerhan's histiocytosis, lymphangitis carcinamatosis, metastatic tumor particularly from vascular cancers like melanoma, renal and thyroid malignancies. Rarely primary lung cancer can also metastasize to lung and appear as miliary opacities. $^{3}$

Lung cancer is the most commonly diagnosed cancer leading to cancer related death worldwide. Here we present a case of 50 year old female patient with Primary lung adenocarcinoma with intrapulmonary miliary opacities.

\section{CASE PRESENTATION:}

A 50-year-old homemaker presented to the hospital with dry cough, low grade fever since 15 days. No history of loss of appetite and loss of weight was noted.

There was no history suggestive of contact with tuberculosis, no significant past medical or surgical history. The patient was not a diabetic or hypertensive.

On examination, patient was alert, awake, moderately built and nourished with no pallor, clubbing or any palpable lymphadenopathy. She was afebrile with regular pulse rate of 105 beats/minute, and blood pressure of $130 / 70 \mathrm{mmHg}$ and respiratory rate of 30 breaths/minute with an oxygen saturation of $92 \% @$ room air. Respiratory system examination revealed bilateral normal vesicular breath sounds in all areas with fine inspiratory creptitations heard over left infra-scapular and infra-axillary areas and there was no abnormality detected on examination of other systems.

Laboratory examination revealed hemoglobin level of $14.3 \mathrm{~g} /$ $\mathrm{dl}$, total leucocyte count of $7000 / \mathrm{mm} 3$ with $72 \%$ neutrophils, $23 \%$ of lymphocytes, $2 \%$ of eosinophils and ESR of $35 \mathrm{~mm} /$ hr. Her liver and renal function tests were within normal range. HIV serology was negative. C-reactive protein was $1.2 \mathrm{mg} / \mathrm{dl}$. CXR PA view showed diffuse nodular opacities involving bilateral lung fields with minimal blunting of left costophrenic angle. HRCT chest showed diffusely and randomly distributed nodular opacities in bilateral lung fields with irregular soft tissue opacity in left lower lobe.

Fiberoptic flexible video bronchoscopy was performed revealing normal cords, Trachea and carina. Bronchoalveolar lavage for cytology showed chronic non specific inflammation and was negative for malignant cells. AFB and CBNAAT were negative. 
Total cell count of BAL fluid was 125 cells/cumm with $10 \%$ neutrophils, $80 \%$ of lymphocytes, $10 \%$ macrophages and Gram's stain ,bacterial culture and sensitivity, $\mathrm{KOH}$ mount for BAL and sputum were negative. Mantoux test was negative. CECT chest showed left lower lobe enhancing lung mass with metastasis involving bilateral lung fields with few lymph nodes in right pretracheal and precarinal region. Histopathological examination of left lower lobe mass showed features suggestive of adenocarcinoma with surrounding insitu component. EGFR 19 deletion mutation was detected on mutation analysis of biopsy specimen. PET CT suggestive of hypermetabolic mass lesion in left lower lung (primary) with mediastinal and multiple small cervical, axillary, abdomino pelvic lymphnodes (metastasis).

The patient was then referred to oncologist and was started on tyrosine kinase inhibitor based therapy.

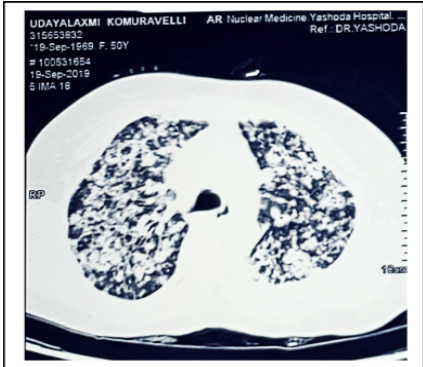

1. Axial image of HRCT Chest showing bilateral miliary opacities with mass in the left lower lobe.

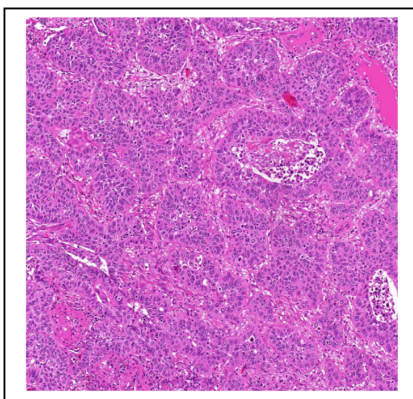

3. HPE Showing features of

Adenocarcinoma

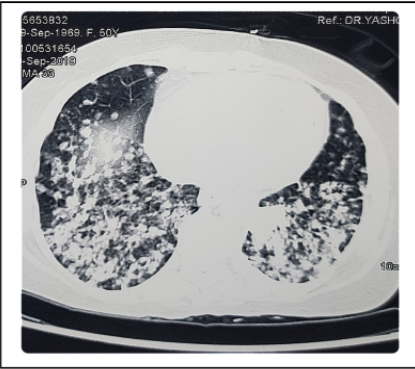

2. Axial image of $\mathrm{CT}$ chest showing left lower lobe lung mass with miliary opacities

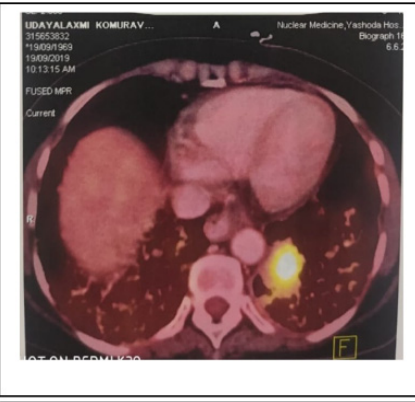

4. PET CT scan showing area of hypermetabolic region in left lower lobe suggestive of malignant mass

\section{DISCUSSION:}

Lung carcinoma is a malignant neoplasm of the lung arising from respiratory epithelium of the bronchus or bronchiole ${ }^{4}$. It is among the most frequently diagnosed cancers and the world's leading cause of cancer related mortality. ${ }^{5}$ The incidence is highest between the age group of 55-65years of age, and active smoking increases the relative risk by thirteenfold and long term passive smoke to cigarette smoke increases by 1.5 fold . ${ }^{4}$ Lung cancers are classified as Small cell and Non small cell lung cancers, these are further divided into three subtypes as squamous cell carcinoma, adenocarcinoma, large cell carcinoma. Pathologically, adenocarcinoma may show acinar, papillary, micropapillary, lepidic or solid growth pattern with either mucin or pneumocyte marker expression and is usually located peripherally with pleural involvement.$^{7}$ Adenocarcinoma is most common primary lung carcinoma accounting for $50 \%$ of cases and the most common lung cancer among non smokers and among women. ${ }^{4}$ Lung cancer predominantly presents with respiratory symptoms as well as symptoms related to obstruction of the airways or adjacent structures. ${ }^{4}$ Miliary spread occurs when tumor cells spread through systemic circulation, get lodged up in the capillary bed and proliferate locally. For intra pulmonary dissemination, rupture into a pulmonary vessel ( arteriole) is the possible cause. ${ }^{4}$ Hematogenous spread can also occur from a metastatic site but in the present case there is no detectable metastatic site on PET Scan.

Lung cancer with miliary metastatic lesions have unfavorable response to treatment and a poor prognosis. ${ }^{8}$

CONCLUSION : Miliary nodules may not always indicate tuberculosis and other possible causes must be explored before starting treatment.

\section{REFERENCES}

1. Richard Webb, Nestor L.Muller, David P.Naidich. HRCT findings:Multiple nodules and Nodular opacities: high resolution CT of the lung, 5 th edition, New Delhi,2015:p.106-107

2. Felson B. The Interstitium. Fundamentals of chest roentgenology. India, 2018, 1;36(1);p.333-334

3. Kimmig L, Bueno J. Miliary nodules: not always tuberculosis. Annals of the American Thoracic Society. 2017 Dec;14(12):1858-60.

4. Pillai S, Khan A, Khan S. Adenocarcinoma of the lung presenting with intrapulmonary miliary metastasis. Cureus. 2019 Aug;11(8).

5. Torre LA, Bray F, Siegel RL, Ferlay J, Lortet-Tieulent J, Jemal A. Global cancer statistics, 2012. CA: a cancer journal for clinicians. 2015 Mar;65(2):87-108

6. Subhashchandra BJ, Ismailkhan M, Shashidhar KC, Narahari MG. A rare case of non-small cell carcinoma of lung presenting as miliary mottling. Iranian journal of medical sciences.2013 Mar;38(1):65.

7. Khan D, Danjuma M, Saddique MU, Murshed KA, Yassin MA. Adenocarcinoma of the Lung Mimicking Miliary Tuberculosis. Case Reports in Oncology. 2020;13(1):13944.

8. Guangsheng P, Weijiao Y, Gang L, Xiaoli W, Lingxia L, Yimin M. Miliary lung adenocarcinoma: a case report. International Journal of Clinical and Experimental Pathology. 2016;9(2):2563-6.

How to cite this article : Vijetha S, Samanvitha V, Ramu M Satish Chandra K, Prasad C N. All that is Miliary is not tuberculosis. Perspectives in Medical Research 2020; 8 (3): $\quad$ 86-87.

DOI: $10.47799 /$ pimr.0803.18

Sources of Support: Nil, Conflict of interest: None declared 